wissen kompakt 2012 $6: 4$

DOI 10.1007/s11838-011-0143-2

(c) Springer-Verlag und Freier Verband Deutscher Zahnärzte e.V. 2012

\section{Wer wir sind und warum wir wissen kompakt herausgeben}

\author{
Die neuen Schriftleiter stellen sich vor
}

Prof. Dr. Dr. Bilal Al-Nawas

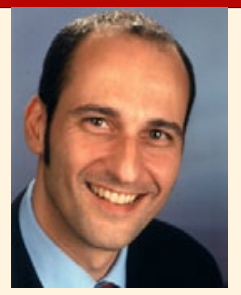

Trotz des scheinbar unbegrenzten Zugangs zu Informationen über das Internet, bleibt es für den Zahnarzt in der Praxis oft schwer, an verlässliche und praxisnahe Übersichtsartikel zu gelangen. Wissenschaftliche Fachzeitschriften publizieren zwar "neuste" Erkenntnisse, sind aber meist in Englisch verfasst und dem Praktiker außerhalb der Universität kaum zugänglich. Daher stellt wissen kompakt für den Leser eine attraktive Quelle für fundierte Übersichtsarbeiten dar und ist für mich als Herausgeber eine Möglichkeit fachliche Nähe zwischen Praxis und Universität herzustellen.

Prof. Dr. Dr. Bilal Al-Nawas, geb. 1968. Studium der Medizin und Zahnmedizin in Frankfurt, Saarbrücken und Zürich mit Promotion 1993 in Zahnmedizin, 1997 in Medizin; 2001 Facharzt für Mund-Kiefer- und Gesichtschirurgie, 2004 Habilitation und Venia legendi im Fach Mund-Kiefer-Gesichtschirurgie; 2005 Zusatzbezeichnung Plastische Operationen; 2006 Preis der Lehre des Fachbereichs Medizin, Universität Mainz; 2009 Gastprofessor an der Kyung Hee University Department of Oral and Maxillofacial Surgery, Seoul, Korea; seit April 2009 W2-Professur für MKG Chirurgie in Mainz (Direktor: Prof. Dr. Dr. W. Wagner). Leitender Oberarzt der Klinik für Mund-, Kieferund Gesichtschirurgie - plastische Operationen, Universitätsmedizin Mainz.

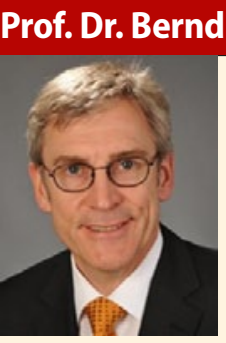

Das Format von wissen kompakt erscheint mir sehr gut dafür geeignet, wissenschaftliche Erkenntnisse in die Praxis zu übertragen. Die Flut neuer Produkte und Technologien, zum Teil aggressiv vermarktet, erfordert eine wissenschaftlich fundierte Bewertung im Hinblick aufihre Praxistauglichkeit. Ich unterstütze das Konzept von wissen kompakt, weil der Zahnarzt verlässliche, neutrale Informationsquellen dieser Art braucht, um seiner Verantwortung bei der Auswahl geeigneter Therapie- und Diagnoseverfahren gerecht zu werden. Hinzu kommt die eigene Freude an der Vermittlung von Wissen.

Prof. Dr. Bernd Haller, geb. 1958 in Trossingen, Studium der Zahnmedizin in Freiburg. Habilitation 1983. 1983-1987 Assistent in der Abteilung für Zahnerhaltung der Universität in Freiburg. 1987-1995 Oberarzt in der Poliklinik für Zahnerhaltung und Parodontologie der Universität Würzburg. 1995 Berufung auf den Lehrstuhl für Zahnerhaltungskunde, Parodontologie und Kinderzahnheilkunde an der Universität Ulm. Geschäftsführender Direktor der Universitätszahnklinik von 1999 bis 2007 und seit 2010. Zwischen 2001 und 2011 Studiendekan für Zahnmedizin.
Prof. Dr. Marc Schmitter

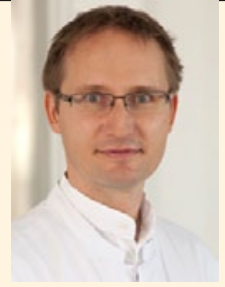

Auch in der Zahnmedizin ist der stetige technische Fortschritt durch innovative Diagnose- und Behandlungsverfahren gerade in der Zeit der "Digitalisierung" vieler Prozessabläufe allgegenwärtig und besonders spannend. Daher freue ich mich über die Möglichkeit, aktuelle wissenschaftliche Ergebnisse im Rahmen dieses traditionsreichen Fortbildungsjournals zusammen mit meinen Kollegen darstellen zu können.

Prof. Dr. Marc Schmitter, 1992-1997 Studium der Zahnmedizin an der Universität Tübingen. 1998-2000 Ausbildungsassistent bei einem niedergelassenen Zahnarzt in Neumünster, 1998 Promotion, 2000 Wissenschaftlicher Mitarbeiter in der Poliklinik für Zahnärztliche Prothetik der Universität Heidelberg, 2004 Ernennung zum Oberarzt, 2005 Spezialist der DGPro, 2006 Habilitation, 2007 Forschungsaufenthalt an der University of Washington, 2008 apl.-Professor. Leitender Oberarzt der Poliklinik für Zahnärztliche Prothetik der Universität Heidelberg, kommissarischer Leiter der Sektion Werkstoffkunde. Spezialist für Funktionsdiagnostik- und Therapie. 